\title{
Dendritic A-Current in Rhythmically Active PreBötzinger Complex Neurons in Organotypic Cultures from Newborn Mice
}

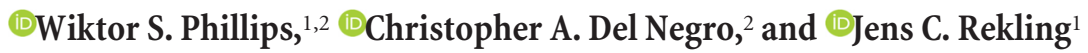 \\ ${ }^{1}$ Department of Neuroscience, University of Copenhagen, DK-2200 Copenhagen N, Denmark, and ${ }^{2}$ Department of Applied Science, Integrated Science \\ Center, The College of William and Mary, Williamsburg, Virginia 23185
}

The brainstem preBötzinger complex (preBötC) generates the inspiratory rhythm for breathing. The onset of neural activity that precipitates the inspiratory phase of the respiratory cycle may depend on the activity of type- 1 preBötC neurons, which exhibit a transient outward $\mathrm{K}^{+}$current, $I_{\mathrm{A}}$. Inspiratory rhythm generation can be studied ex vivo because the preBötC remains rhythmically active in vitro, both in acute brainstem slices and organotypic cultures. Advantageous optical conditions in organotypic slice cultures from newborn mice of either sex allowed us to investigate how $I_{\mathrm{A}}$ impacts $\mathrm{Ca}^{2+}$ transients occurring in the dendrites of rhythmically active type-1 preBötC neurons. The amplitude of dendritic $\mathrm{Ca}^{2+}$ transients evoked via voltage increases originating from the soma significantly increased after an $I_{\mathrm{A}}$ antagonist, 4-aminopyridine (4-AP), was applied to the perfusion bath or to local dendritic regions. Similarly, glutamate-evoked postsynaptic depolarizations recorded at the soma increased in amplitude when 4-AP was coapplied with glutamate at distal dendritic locations. We conclude that $\mathrm{I}_{\mathrm{A}}$ is expressed on type-1 preBötC neuron dendrites. We propose that $I_{\mathrm{A}}$ filters synaptic input, shunting sparse excitation, while enabling temporally summated events to pass more readily as a result of $I_{\mathrm{A}}$ inactivation. Dendritic $I_{\mathrm{A}}$ in rhythmically active preBötC neurons could thus ensure that inspiratory motor activity does not occur until excitatory synaptic drive is synchronized and well coordinated among cellular constituents of the preBötC during inspiratory rhythmogenesis. The biophysical properties of dendritic $I_{\mathrm{A}}$ might thus promote robustness and regularity of breathing rhythms.

Key words: A-current; breathing; dendrites; potassium channels; preBötzinger; respiratory neurons

\section{Significance Statement}

Brainstem neurons in the preBötC generate the oscillatory activity that underlies breathing. PreBötC neurons express voltagedependent currents that can influence inspiratory activity, among which is a transient potassium current $\left(I_{\mathrm{A}}\right)$ previously identified in a rhythmogenic excitatory subset of type-1 preBötC neurons. We sought to determine whether $I_{\mathrm{A}}$ is expressed in the dendrites of preBötC. We found that dendrites of type-1 preBötC neurons indeed express $I_{\mathrm{A}}$, which may aid in shunting sparse non-summating synaptic inputs, while enabling strong summating excitatory inputs to readily pass and thus influence somatic membrane potential trajectory. The subcellular distribution of $I_{\mathrm{A}}$ in rhythmically active neurons of the preBötC may thus be critical for producing well coordinated ensemble activity during inspiratory burst formation.

\section{Introduction}

Bilaterally distributed in the ventrolateral medulla, the preBötC contains a network of excitatory interneurons that generate the

\footnotetext{
Received Nov. 26, 2017; revised Jan. 22, 2018; accepted Feb. 9, 2018.

Author contributions: W.S.P., C.A.D.N., and J.C.R. designed research; W.S.P. and J.C.R. performed research; W.S.P., C.A.D.N., and J.C.R. analyzed data; W.S.P., C.A.D.N., and J.C.R. wrote the paper.

This work was supported by The Danish Research Council, The Lundbeck Foundation, Mindefonden for Alice Brenaa, Den Owensenke Fond, Lægeforeningens forskningsfond, Agnes og Pouls Friis Fond, Fonden til Lægevidenskabens Fremme, Brødrene Hartmans fond, and the U.S. National Institutes of Health (R01 HL104127 PI to C.A.D.N.). We thank Morten Bjerre Nielsen for technical assistance.

The authors declare no competing financial interests.

Correspondence should be addressed to Dr. Jens C. Rekling, Department of Neuroscience, University of Copenhagen, Panum Building 33.3.84, Blegdamsvej 3, DK-2200 Copenhagen N, Denmark. E-mail: jrekling@sund.ku.dk.
}

rhythm for inspiratory breathing movements (Smith et al., 1991; Feldman and Del Negro, 2006; Feldman et al., 2013). PreBötC neurons can be subdivided into two classes, which differ by their electroresponsive properties and membrane potential trajectory during the respiratory cycle (Rekling et al., 1996; Picardo et al., 2013). During rhythmic activity, type-1 preBötC neurons integrate synaptic drive and exhibit a ramp of preinspiratory depolarization $\sim 400 \mathrm{~ms}$ before inspiratory bursts. Type-2 neurons exhibit preinspiratory depolarization as well, but it occurs $\sim 200 \mathrm{~ms}$ 
later than type-1 neurons (Rekling et al., 1996). Earlier preinspiratory activity in type-1 neurons suggests that they may be more important for initiating the cascade of recurrent excitation that leads to synchronized network bursts (Smith et al., 1990; Rekling et al., 1996; Carroll and Ramirez, 2013; Kam et al., 2013; Feldman and Kam, 2015). Type-1 neurons are thus putatively rhythmogenic.

The biophysical basis by which type-1 neurons synchronize and coordinate the onset of activity across the rhythmic neuronal population has yet to be fully explained, but may be related to the presence of a transient outward $I_{\mathrm{A}}$-like current (Rekling et al., 1996). Pharmacological inhibition of $I_{\mathrm{A}}$ in acute slices containing the preBötC results in spurious burst generation at both the cellular and network levels. Such changes in rhythmic activity have been attributed to disorderly recruitment of rhythmogenic preBötC neurons during recurrent excitation, which precedes the inspiratory burst in each cycle of the rhythm (Hayes et al., 2008).

$I_{\mathrm{A}}$ can influence synaptic integration, as shown in principal neurons outside of the preBötC. For example, $I_{\mathrm{A}}$ is expressed at a high density on the dendrites of hippocampal pyramidal cells and cerebellar Purkinje cells, where it acts locally to inhibit $\mathrm{Ca}^{2+}$ spikes and limits the effect of sparse or transient excitatory inputs (Hoffman et al., 1997; Kampa and Stuart, 2006; Otsu et al., 2014). Conversely, $I_{\mathrm{A}}$ on dendrites permits robust postsynaptic responses to temporally summated excitatory input, which may be long-lasting enough to inactivate $I_{\mathrm{A}}$ and thereby depolarize the membrane (Magee et al., 1998).

Dendrites of rhythmically active preBötC neurons feature active integrative properties, but the dendritic expression of inward and outward currents in subclasses of preBötC neurons is presently unclear. The amplitude of the synaptic drive potential underlying inspiratory bursts in rhythmically active preBötC neurons depends on a $\mathrm{Ca}^{2+}$-activated nonspecific cation current $\left(I_{\text {CAN }}\right)$, recruited by concurrent $\mathrm{Ca}^{2+}$ influx through voltage-gated channels or intracellular $\mathrm{Ca}^{2+}$ release linked to group I metabotropic glutamate receptors (mGluRs; Pace et al., 2007; Mironov, 2008; Pace and Del Negro, 2008; Mironov and Skorova, 2011). Excitatory potentials recorded at the soma increase when group I mGluR agonists are coapplied with AMPA on dendrites (Pace and Del Negro, 2008), suggesting that intrinsic dendritic conductances can locally amplify synaptic input. Spontaneous synaptic activity during the interval between inspiratory bursts could cause spurious burst generation if that activity were to evoke $I_{\text {CAN }}$ on its own. Therefore, a biophysical mechanism that selectively inhibits sparse synaptic inputs could prevent aberrant dendritic amplification, thus avoiding spurious bursts before a synchronized build-up in network activity.

Fast-activating voltage-dependent outward currents on dendrites can shunt sparse excitatory input, and might explain the disordered behavior seen during network-wide blockade of $I_{\mathrm{A}}$ in rhythmically active slices. We therefore hypothesize that the blockade of $I_{\mathrm{A}}$ modifies active synaptic integration occurring in the dendrites in type-1 neurons. Here, we use an organotypic slice culture containing the preBötC to investigate how $I_{\mathrm{A}}$ might influence transient depolarization in the dendrites of rhythmically active preBötC neurons. We show that loss of dendritically localized $I_{\mathrm{A}}$ significantly increases the amplitude of voltage-sensitive dendritic $\mathrm{Ca}^{2+}$ transients as well as glutamate-evoked postsynaptic depolarizations recorded at the soma. These phenomena demonstrate a possible mechanism by which dendritic $I_{\mathrm{A}}$ might filter inputs and thus modulate the postsynaptic output of type-1 neurons, blocking sparse synaptic excitation while permitting temporally and spatially summated inputs during the preinspira- tory phase of the respiratory cycle, which may enhance the regularity of inspiratory rhythms.

\section{Materials and Methods}

Ethical approval. The Department of Experimental Medicine at the Faculty of Health and Medical Sciences, University of Copenhagen, approved all experiments and procedures according to protocols laid out by Danish Ministry of Justice and the Danish National Committee for Ethics in Animal Research.

Organotypic slice cultures. US Naval Medical Research Institute (NMRI) mice postnatal day (P) 3.5 to P6.5 of either sex were anesthetized with isoflurane (Baxter) and immediately dissected in sterile-filtered chilled artificial CSF (ACSF) containing the following (in mM): 184 glycerol, $2.5 \mathrm{KCl}, 1.2 \mathrm{NaH}_{2} \mathrm{PO}_{4}, 30 \mathrm{NaHCO}_{3}, 5$ HEPES acid, 15 HEPES base, 25 D-glucose, 5 sodium ascorbate, 2 thiourea, 3 sodium pyruvate, 10 $\mathrm{MgSO}_{4}, 0.5 \mathrm{CaCl}_{2}, \mathrm{pH} 7.3$, equilibrated by bubbling with $95 \% \mathrm{O}_{2} / 5 \%$ $\mathrm{CO}_{2}$. A single transverse slice of the brainstem, $400 \mu \mathrm{m}$ in thickness, were taken at the level of the preBötC using a vibrating microtome (ThermoFisher Scientific Microm 650V; RRID:SCR_008452). Anatomical markers, such as the principle loop of the inferior olive and obex of the fourth ventricle (Ruangkittisakul et al., 2011, 2014), were used to verify the rostral-caudal location of the preBötC and thus properly calibrate the slice. Cultures were prepared as previously described (Phillips et al., 2016) via the Stoppini interface method, placing 1-4 transverse brainstem slices onto semi-porous culture well inserts (Millipore, Catalog \#PIC03050; RRID:SCR_008983). Mounted preparations were maintained in sterile-filtered organotypic culture media containing the following: 50\% Eagle's MEM with Earle's salts, 25\% HBSS, 25\% horse serum, $2 \mathrm{~mm}$ GlutaMAX (Invitrogen), $200 \mathrm{U} / \mathrm{ml}$ penicillin, $5 \mu \mathrm{g} / \mathrm{ml}$ streptomycin, $25 \mathrm{~mm}$ HEPES, and an additional $3.6 \mathrm{~mm}$ D-glucose. The osmolarity of the culture medium measured 320-340 mOsm with $\mathrm{pH}$ 7.25. The cultures were treated with $10 \mu \mathrm{M}$ MK- 801 for the first $3 \mathrm{~d}$ in vitro to prevent ischemia-related cell death (Newell et al., 1990). Fresh culture media was supplied every $48 \mathrm{~h}$ thereafter until experimentation. These slices were then kept in a sterile, humidified incubator at $35^{\circ} \mathrm{C}$ and atmospheric $\mathrm{CO}_{2}$ concentrations during incubation. All compounds and media were from Sigma-Aldrich (RRID:SCR_008988).

Electrophysiology. Somatic whole-cell patch-clamp recordings were performed in current-clamp and discontinuous single-electrode voltageclamp (dSEVC; sampling rate $1-2 \mathrm{kHz}$ ) using an AxoClamp 2B amplifier (Molecular Devices). Data were digitally acquired at a sampling rate of 10 $\mathrm{kHz}$, with a low-pass filter of $2 \mathrm{kHz}$ from the output of the AxoClamp 2B. Glass micropipettes were pulled from filamented capillary glass [outer diameter (o.d.) $1.5 \mathrm{~mm}$, inner diameter (i.d.) $0.86 \mathrm{~mm}$; Harvard Apparatus] using a PUL-100 micropipette puller (World Precision Instruments; RRID:SCR_008593) to a tip resistance of 4-6 $\mathrm{M} \Omega$. Patch pipettes were filled with a solution containing the following (in mM): $130 \mathrm{HCH}_{3} \mathrm{SO}_{3}, 130$ $\mathrm{KOH}, 10$ HEPES, $0.4 \mathrm{NaGTP}, 4 \mathrm{Na}_{2} \mathrm{ATP}, 5 \mathrm{Na}_{2}$-phosphocreatine, $4 \mathrm{MgCl}_{2}$ (all from Sigma-Aldrich; RRID:SCR_008988), 0.05 AlexaFluor 594 hydrazide (FisherThermo Scientific; RRID:SCR_008452), 0.1 Fluo-8L (AAT Bioquest). The osmolarity of the patch pipette solution measured $310 \mathrm{mOsm}$ with a $\mathrm{pH}$ of 7.3. Patch pipettes were visually guided to target neurons under visual control using MPC-200 micromanipulator system (Sutter Instruments) on a fixed-stage upright microscope (modified Olympus BX51) under $40 \times$ magnification $(\mathrm{NA}=0.8, \mathrm{WD}=3.3 \mathrm{~mm})$.

Iontophoresis was performed using a IP-X5 instrument (Neuro Data Instruments). Single-barrel glass micropipettes were pulled as described above to a tip resistance of $8-10 \mathrm{M} \Omega$ for single-drug trials. For experiments involving 4-aminopyridine (4-AP), pipettes were filled with an aqueous solution containing the following: $165 \mathrm{~mm} \mathrm{NaCl}, 10 \mathrm{~mm}$ HEPES, and $0.2 \%$ tetramethylrhodamine-dextran. For inhibition of $I_{\mathrm{A}}$ localized to dendrites, we added $45 \mathrm{~mm}$ 4-AP, $\mathrm{pH}$ 7.5. For experiments involving local iontophoretic application of $\mathrm{Cd}^{2+}$, an aqueous solution containing exclusively $200 \mathrm{mM} \mathrm{CdSO}_{4}$ was used. ACSF in $\mathrm{Cd}^{2+}$ experiments was free of phosphates (omitting $\mathrm{NaH}_{2} \mathrm{PO}_{4}$ ) to avoid precipitation. Pulses were delivered at $1 \mathrm{~Hz}(500 \mathrm{~ms}$ pulse duration) with the ejection currents: $+20-30 \mathrm{nA}$ to eject $4-\mathrm{AP},+5-10 \mathrm{nA}$ to eject $\mathrm{Cd}^{2+}$. Filamented theta capillary glass (o.d. $1.5 \mathrm{~mm}$, i.d. 1.17, septum $0.17 \mathrm{~mm}$; Harvard Apparatus) was used in some experiments to apply glutamate (150 mM in 
water, $\mathrm{pH} 8,+20 \mathrm{nA}$ holding, $-150 \mathrm{nA}$ ejection current, $0.2 \mathrm{~Hz}$ cycle time, $100 \mathrm{~ms}$ pulse duration) from one barrel and 4-AP (50 mM with $10 \mathrm{~mm}$ HEPES in $165 \mathrm{~mm} \mathrm{NaCl}, \mathrm{pH} 7.5,-10 \mathrm{nA}$ holding, $+90 \mathrm{nA}$ ejection current, 10-30 s per application bout) from the other.

During voltage-clamp recordings and dendritic glutamate iontophoresis, $1 \mu \mathrm{M}$ TTX (Tocris Bioscience; RRID:SCR_003689) was added to the bath. A-current was blocked with bath-applied $2 \mathrm{~mm}$ 4-AP when not applied locally. During spike train response experiments, $10 \mu \mathrm{M}$ NBQX disodium salt (Tocris Bioscience; RRID:SCR_003689) was added to the bath to preclude $\mathrm{Ca}^{2+}$ transients that might arise due to spontaneous excitatory synaptic transmission.

Rhythmic activity in slices was recorded in ACSF containing the following (in mM): $124 \mathrm{NaCl}, 3 \mathrm{KCl}, 5 \mathrm{KH}_{2} \mathrm{PO}_{4}, 25 \mathrm{NaHCO}_{3}, 25$ D-glucose, 1 ascorbic acid, $1 \mathrm{MgCl}_{2}, 1.5 \mathrm{CaCl}_{2}$ (all from Sigma-Aldrich; RRID: SCR_008988), with a pH of 7.4, equilibrated by bubbling with $95 \%$ $\mathrm{O}_{2} / 5 \% \mathrm{CO}_{2}$. The final concentration of $\mathrm{K}^{+}$was $\left[\mathrm{K}^{+}\right]_{\mathrm{o}}=8 \mathrm{~mm}$, which elevates baseline membrane potentials and increases the frequency of spontaneous respiratory rhythm.

After recording over 15 min of rhythmic activity, the standard ACSF was always exchanged with a low-excitability ACSF in which external $\mathrm{K}^{+}$ concentration was reduced from 8 to $3 \mathrm{~mm}$ and external $\mathrm{Ca}^{2+}$ was raised from 1.5 to $2 \mathrm{~mm}$. Thus, all subsequent electrophysiological measurements and subcellular imaging were performed at physiological $\mathrm{K}^{+}$concentrations. These modifications reduced or stopped network rhythmic activity by hyperpolarizing neuronal membrane potentials and increasing the threshold for $\mathrm{Na}^{+}$channel activation (Panaitescu et al., 2009; Ruangkittisakul et al., 2011). Low-excitability ACSF contained the following (in mM): $124 \mathrm{NaCl}, 2.5 \mathrm{KCl}, 1.25 \mathrm{NaH}_{2} \mathrm{PO}_{4}, 25 \mathrm{NaHCO}_{3}, 25$ D-glucose, 1 ascorbic acid, $1 \mathrm{MgCl}_{2}, 2 \mathrm{CaCl}_{2}$, with a $\mathrm{pH}$ of 7.4 , equilibrated by bubbling with $95 \% \mathrm{O}_{2} / 5 \% \mathrm{CO}_{2}$.

$\mathrm{Ca}^{2+}$ imaging. The region of the organotypic culture containing the preBötC was targeted via anatomical landmarks and visualization of synchronized rhythmic network activity during whole-slice $\mathrm{Ca}^{2+}$ imaging (Phillips et al., 2016). A membrane-permeable fluorescent $\mathrm{Ca}^{2+}$ indicator, either Fura-2 AM or Fluo-8, AM (AAT Bioquest), was bath loaded before each experiment. Cultures were submerged in $1.5 \mathrm{ml}$ of loading solution containing $35 \mu \mathrm{M}$ of Fura-2 AM or Fluo-8, AM in DMSO along with $0.05 \%$ Pluronic acid dissolved in low excitability ACSF. Slices were incubated in loading solution at room temperature for 30-40 $\mathrm{min}$, and then perfused with ACSF in the recording chamber for 15 min of recovery and additional de-esterification before experimentation. Preheated and oxygenated ACSF was perfused into the recording chamber during recording at $2 \mathrm{ml} / \mathrm{min}$ and $29^{\circ} \mathrm{C}$.

Fluorescent $\mathrm{Ca}^{2+}$ activity was recorded in wide-field on a fixed-stage upright microscope (modified Olympus BX51), illuminated by a metal halide light source PhotoFluor II (89North) or a blue (470 nm) LED light source, M470L2 (Thorlabs). Red and green channel fluorescence was visualized using a dual-bandpass filter set (Chroma 59022: excitation dual bandpass 450 - 490/555-590 nm, emission dual bandpass 500-543/ 603-665 nm). Red and green channels (for AlexaFluor 594 hydrazide, Fluo-8, AM, Fluo-8L) were separated during acquisition by manually exchanging an additional excitation filter in the light path (Semrock FF01: bandpass 565-605 nm; Semrock FF02: bandpass 457-487 nm). Imaging of Fura-2 AM loaded cultures was done using a U-MNU filter [Olympus, UV Narrow, Excitation: 360-370 nm, dichroic mirror: 400 $\mathrm{nm}$ (long-pass), emission: $420 \mathrm{~nm}$ (long-pass)]. Time series acquisition was performed with a sCMOS camera (Neo DC-152Q, Andor Technology) controlled by SOLIS software (Andor Technology). Imaging protocols used $10 \times($ NA 0.3$)$ and $40 \times($ NA 0.8$)$ water-immersion objectives. Time series were acquired at $10-50 \mathrm{~Hz}$. At least $30 \mathrm{~min}$ of perfusion was allowed after break-in during whole-cell patch-clamp to allow equilibration of intracellular $\mathrm{Ca}^{2+}$ dye. Dark current noise, the background sensor noise in the absence of illumination, was sampled before the start of each acquisition and subtracted from all imaging sweeps. In some experiments, $40 \mathrm{~nm}$ pandinotoxin-K $\alpha$ (Sigma-Aldrich; RRID:SCR_008988), $500 \mathrm{~nm}$ phrixotoxin-1 or $100 \mathrm{~nm}$ AmmTX3 (Alomone Labs; RRID: SCR_013570), which are $I_{\mathrm{A}}$-blockers that act selectively on Kv4.x channel subtypes, were applied to the perfusion bath.
Experimental design and statistical analysis. Optical data were analyzed off-line using ImageJ 1.51p (RRID:SCR_003070), and Igor Pro 7 (RRID: SCR_000325). Dendritic $\mathrm{Ca}^{2+}$ transients were calculated as the percentage change in fluorescence relative to baseline values $\left(\Delta F / F_{0}\right)$. Background was subtracted frame-by-frame, taken as the equal-sized mean background fluorescence immediately adjacent to the dendritic ROI. Dendritic ROIs were hand drawn along neural processes, extending for $\leq 10$ $\mu \mathrm{m}$ in length. The mean prestimulus fluorescence in the dendritic ROI was defined as $F_{0}$. For each condition, $4-6$ sweeps were acquired at $10-30 \mathrm{~s}$ intervals, and subsequently averaged after calculation of $\Delta F / F_{0}$.

Subcellular differences in the concentration of the $\mathrm{Ca}^{2+}$ indicator, surface area to volume ratio, and $\mathrm{Ca}^{2+}$ channel density can alter the amplitude of $\Delta F / F_{0}$ in response to a given voltage increase (Yasuda et al., 2004). These experiments seek to measure relative changes in membrane excitability caused by manipulations of $I_{\mathrm{A}}$, but not the absolute change in $\mathrm{Ca}^{2+}$ concentration. The changes to $\mathrm{Ca}^{2+}$ transients before and after 4-AP application were therefore also computed at each measurement location to control for such differences between cellular compartments or between experiments that might bias the weight of fluorescence measurements. The formula for this metric, which we term normalized 4-AP effect, was the difference (i.e., before and after blockade 4-AP) in $\mathrm{Ca}^{2+}$ transient amplitude in response to stimuli divided by the sum of the responses:

$$
\frac{\text { Amplitude After - Amplitude Before }}{\text { Amplitude After }+ \text { Amplitude Before }}
$$

Electrophysiological data were acquired using pClamp 10.0 (RRID: SCR_011323) and subsequently analyzed using custom scripts written in Igor Pro 7 (RRID:SCR_000325).

Statistical values are reported as mean $\pm \mathrm{SD}$, unless otherwise stated. Student's $t$ test was used for statistical comparisons of one- and twosample populations. Fisher's exact test was used to assess the distribution of neuronal membrane currents. All statistical tests were performed using OriginPro 2017 (Microcal; RRID:SCR_002815) or Prism 7 (GraphPad; RRID:SCR_002798).

\section{Results}

Effects of low-dose 4-AP on rhythmic bursting in organotypic cultures of the preBötC

Brainstem organotypic slice cultures containing the preBötC generate spontaneous bilaterally synchronized rhythmic bursts of ensemble neuronal activity resembling inspiratory rhythms analogous generated by acutely prepared slices (Phillips et al., 2016), which can be recorded via population fluorescent $\mathrm{Ca}^{2+}$ activity. If $I_{\mathrm{A}}$ moderates postsynaptic amplification of excitatory input, then partial inhibition of $I_{\mathrm{A}}$ ought to lower the threshold for postsynaptic burst production, thereby advancing inspiratory phase onset, which we surmise would measurably increase rhythmic frequency. We imaged rhythmic cultures and found that bath-applied low doses of the $I_{\mathrm{A}}$-blocker 4-AP (50 $\left.\mu \mathrm{M}\right)$ increased the oscillatory burst frequency observed in the preBötC from $13.6 \pm 2.7$ to $24.3 \pm 5.1$ bursts $/ \mathrm{min}$ (Fig. $1 A ; n=7$ cultures, $t_{(6)}=$ $4.68, p=0.0034$, paired two-tailed $t$ test).

In addition to its affinity for potassium channels of the $\mathrm{Kv} 4$ family (associated with $I_{\mathrm{A}}$-like currents), 4-AP can also block delayed rectifier Kv1.1 and Kv1.2 channels, which are typically expressed in axons. We thus attempted to pharmacologically isolate $I_{\mathrm{A}}$-related effects via bath application of three scorpion and spider toxin $I_{\mathrm{A}}$-blockers that have greater, albeit heterogenous selectivity for the Kv4 channel subfamily, i.e., pandinotoxin-K $\alpha$, phrixotoxin-1, or AmmTX3 (Diochot et al., 1999; Klenk et al., 2000; Maffie et al., 2013). However, none of these selective blockers changed burst frequency (Fig. $1 A ; n=3$ cultures in each group; $F_{(1,2)}=2.62, p=0.24$, repeated-measures one-way ANOVA). 
Low doses of 4-AP also affected the relative amplitude of synchronized $\mathrm{Ca}^{2+}$ activity in regions of the slice putatively corresponding to downstream motor and sensory nuclei (i.e., hypoglossal motor nucleus and the nucleus of the solitary tract). The amplitude of synchronized $\mathrm{Ca}^{2+}$ transients located dorsomedial to the preBötC was increased to $190 \pm 55 \%$ in response to low-doses of 4-AP (Fig. 1B; calculated as the percentage change in the ratio of dorsomedial region: preBötC $\mathrm{Ca}^{2+}$ transient amplitudes for equal-sized areas, $n=8$ cultures; $t_{(7)}=5.83, p=0.0006$, two-tailed paired $t$ test).

We took advantage of the improved optical conditions in the cultures and sampled 10 somatic ROIs within the preBötC, as well as a pooled sample of dendritic ROIs in the neighboring neuropil (area: $4000-5000 \mu \mathrm{m}^{2}$ ) imaged in a single field of view, before and after bath application of 4-AP (Fig. 1C,D). Baseline rhythmic $\mathrm{Ca}^{2+}$ transients measured in dendrites of the neuropil had a shorter duration (halfamplitude width: $672 \pm 188 \mathrm{~ms}$ ) than those arising in somatic compartments (955 \pm $262 \mathrm{~ms}, n=7$ cultures, $t_{(6)}=7.53, p=$ 0.0003 , two-tailed paired $t$ test). In the presence of 4-AP the half-amplitude width and decay time constant of the $\mathrm{Ca}^{2+}$ transients increased in both somatic ROIs (half-amplitude width: $955 \pm 262$ ms vs $1181 \pm 416 \mathrm{~ms}$; decay time constant: $0.96 \pm 0.19 \mathrm{~s}$ vs $1.73 \pm 0.77 \mathrm{~s} ; n=7$ cultures, $t_{(6)}=3.30, p=0.0163, t_{(6)}=$ $2.82, p=0.030$, two-tailed paired $t$ test) and neuropil dendritic ROIs (half-amplitude width: $672 \pm 188 \mathrm{~ms}$ vs $929 \pm 316$ ms; decay time constant: $0.84 \pm 0.85 \mathrm{~s}$ vs $1.72 \pm 1.07 \mathrm{~s} ; n=7$ cultures, $t_{(6)}=4.34$, $p=0.0049, t_{(6)}=2.68, p=0.037$, twotailed paired $t$ test; Fig. 1E). The mean rise-time did not change significantly (somata: $338 \pm 68$ vs $344 \pm 103, n=7$ cultures, $t_{(6)}=0.29, p=0.7769$; neuropil dendrites: $248 \pm 53$ vs $278 \pm 121, n=7$ cultures, $t_{(6)}=0.54, p=$ 0.6092 , two-tailed paired $t$ test). These data suggest that 4-AP increases network excitability. To determine whether this increased excitability might be attributable to cellular-level changes in synaptic integration, we investigated the subcellular expression of $I_{\mathrm{A}}$ in a class of neurons thought to be most critical to rhythm generation.

\section{Two rhythmic neuron classes in the preBötC}

Organotypic slice cultures containing the preBötC generate respiratory rhythm analogous to acute slice preparations and en bloc brainstem-spinal cord preparations (Funk and Greer, 2013; Forsberg et al., 2016; Phillips et al., 2016). However, it is not yet known whether rhythmically active preBötC neurons in culture retain the dichotomous membrane properties classified as type-1 and type-2 first characterized in acute slice recordings (Rekling et al., 1996). Type- 1 and type- 2 neurons are distinguished by volt-
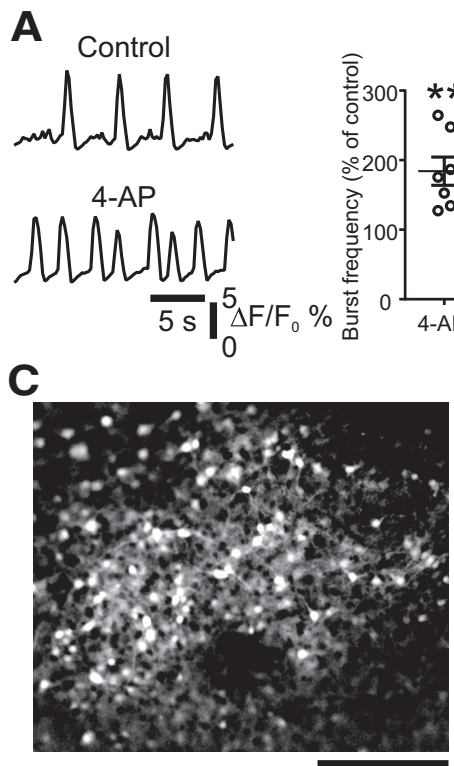

E

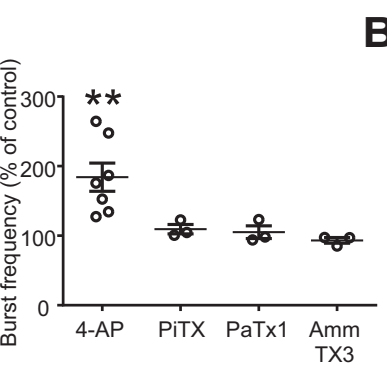

B

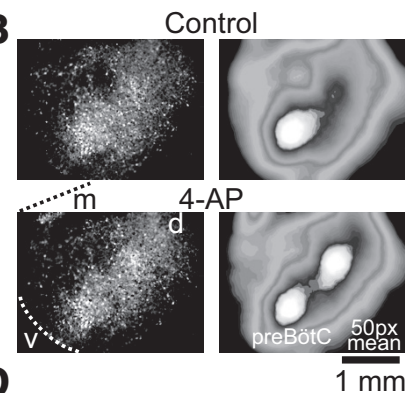

Figure 1. 4-AP increases burst frequency, dorsomedial burst amplitude, and duration of soma and dendritic $\mathrm{Ca}^{2+}$ transients. $A$, Left, $\mathrm{Ca}^{2+}$ transients in a Fluo-8, AM loaded slice culture before (Control) and after bath applied 4-AP (50 $\left.\mu \mathrm{M}\right)$. Right, Group data for bath applied I/Alockers 4-AP (50 $\mu \mathrm{m} ; n=7)$, pandinotoxin-K $\alpha(\mathrm{PiTX} ; 40 \mathrm{~nm} ; n=3)$, phrixotoxin-1 (PaTx1; $500 \mathrm{~nm} ; n=3)$, wing grayscale-coded normalized (min: black, max: white) averages. M, Midline; $v$, ventral; $d$, dorsal. Note that 4-AP induces a (bottom right), but that this type of non-ratiometric AM dye-based $\mathrm{Ca}^{2}$ 列 over 10 somatas (circles) and the neuropil (black filled areas). D, Left, Blow-up of region defined by the dotted line in $\mathbf{C}$ (right) signal (F) from the 10 somas and neuropil, before (Cont) and after 4-AP (50 $\mu \mathrm{m}, 4-\mathrm{AP})$. Note the increase in duration of the $\mathrm{Ca}^{2+}$ signal in both soma and dendrite regions. $\boldsymbol{E}$, Group data of the half-amplitude width, decay time constant (TauM), and rise time $10-90 \%$ for raw burst $\mathrm{Ca}^{2+}$ signals in soma and dendritic regions before and after 4-AP $(50 \mu \mathrm{m} ; n=7)$. Note an increase in width, TauM, but not rise time in response to $4-\mathrm{AP} .\left({ }^{*} p<0.05,{ }^{* *} p<0.01,{ }^{* * *} p<0.001\right)$

age trajectory (e.g., preinspiratory activity) and the expression of either a transient outward current, $I_{\mathrm{A}}$, or a hyperpolarizationactivated inward current, $I_{\mathrm{h}}$. Type-1 neurons express $I_{\mathrm{A}}$, lack $I_{\mathrm{h}}$, and display a ramp-like increase in membrane potential, known as preinspiratory activity, beginning $\sim 400 \mathrm{~ms}$ before the inspiratory burst. Conversely, type- 2 neurons lack $I_{\mathrm{A}}$, express $I_{\mathrm{h}}$, and display more latent preinspiratory activity $\sim 200 \mathrm{~ms}$ before the inspiratory burst (Rekling et al., 1996). Motor nerve rootlets are not retained in slice cultures, so here type- 1 and type- 2 neurons were differentiated based on the presence of either $I_{\mathrm{A}}$ or $I_{\mathrm{h}}$, but not preinspiratory latency.

We recorded rhythmically active neurons in organotypic cultures and tested for the expression of both $I_{\mathrm{A}}$ and $I_{\mathrm{h}}$ in currentclamp upon break-in in the whole-cell configuration (Fig. $2 ; n=$ 42). Rhythmic activity was first recorded using the minimum amount of negative holding current to inhibit spontaneous action potentials between rhythmic bursts (Fig. $2 A ; 0$ to $-0.15 \mathrm{nA}$ ). 
A Type-1

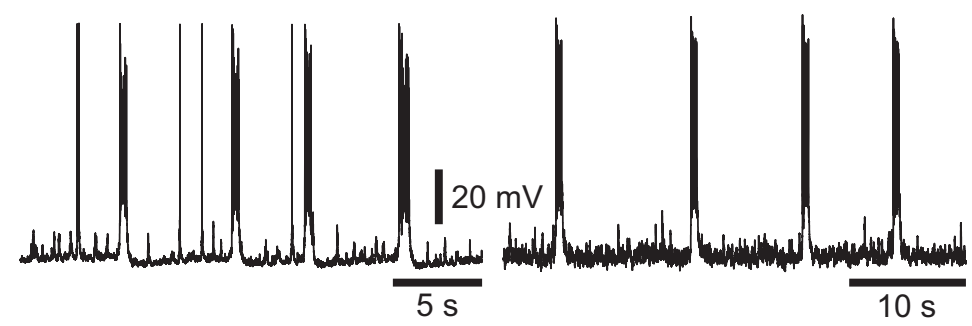

B
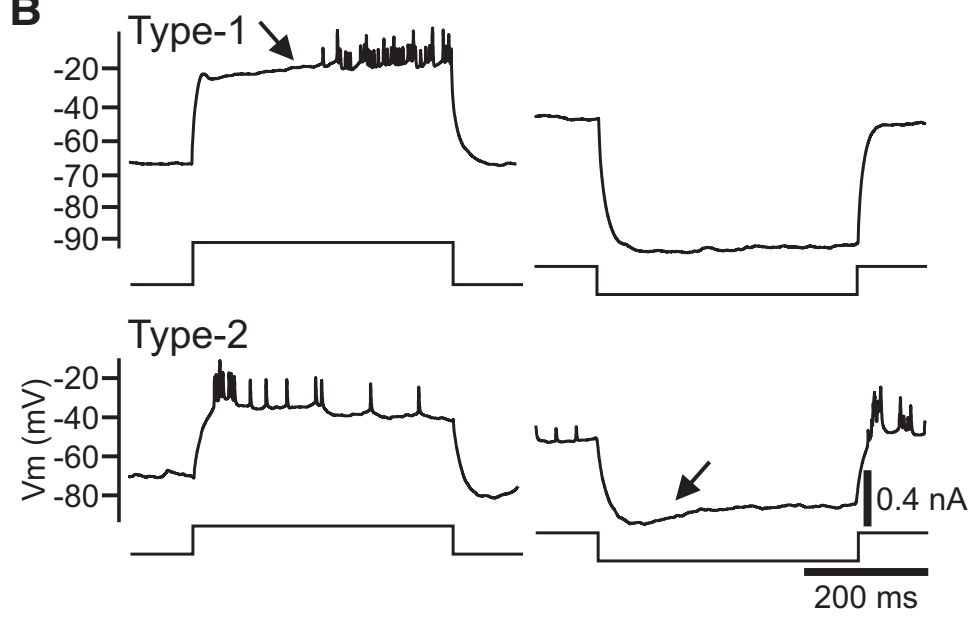

Figure 2. Electroresponsive properties of oscillating type-1 and type-2 neurons. $\boldsymbol{A}$, Spontaneous oscillatory burst activity in two neurons recorded in current camp mode. Note that the burst in the left-most neuron show afterhyperpolarizations following the bursts. $\boldsymbol{B}$, Depolarizing and hyperpolarizing square current pulses from a slightly hyperpolarized membrane potential, and around resting $V_{m}$, give rise to two distinctive electroresponsive responses in the two neurons. The type- 1 neuron show delayed excitation (arrow, top left trace), and the type-2 neuron show a sag potential (arrow, bottom right). Voltage traces are cycle-triggered averages of 5-10 sweeps, which truncates action potentials but retains the form of delayed excitation and sag potentials.

neurons. However, $7 \%(n=3)$ of the neurons showed both sag potential and delayed excitation and 5\% $(n=2)$ displayed neither property, so these neurons $(n=5)$ could not be categorized as either type- 1 or -2 . These results are in line with the distribution of respiratory neuron classes in acute slices (Rekling et al., 1996; Picardo et al., 2013). If we consider the null hypothesis to be that there is no relationship governing the expression of $I_{\mathrm{A}}$ and $I_{\mathrm{h}}$ in respiratory neurons, then the allotment of recorded neurons into type- 1 and type- 2 phenotypes is unlikely to have occurred by random chance $(p=0.0001$, Fisher's exact test), suggesting that the dichotomous electrophysiological properties of rhythmically active type- 1 and type-2 neurons observed in the preBötC from acute slices, also persists in culture.

\section{Voltage ramps during blockade of $I_{\mathrm{A}}$ increase electrotonic compactness of type-1 neurons}

To determine whether $I_{\mathrm{A}}$ might actively inhibit the spread of voltage transients along dendrites in type-1 neurons, we next performed simultaneous SEVC and fluorescent $\mathrm{Ca}^{2+}$ imaging of rhythmically active neurons (Fig. 3). This allowed us to track the relative amplitude of voltage changes at distal dendritic compartments using voltage-sensitive $\mathrm{Ca}^{2+}$ influx as a surrogate for direct measurements of membrane potential.

Among all rhythmically active neurons, the mean interburst interval was $5.4 \pm 3.2 \mathrm{~s}$, and the mean burst duration was $405 \pm 134$ $\mathrm{ms}(n=42)$.

The presence of $I_{\mathrm{A}}$ was determined by first hyperpolarizing neurons with negative holding current to a baseline membrane potential $\left(V_{\mathrm{m}}\right)$ lower than $-65 \mathrm{mV}$, which de-inactivates $I_{\mathrm{A}}$ (Hayes et al., 2008). Square-wave-positive current pulses of 400 $\mathrm{ms}$ duration were then delivered to evoke repetitive firing of action potentials. Neurons with $I_{\mathrm{A}}$ display a delay in membrane depolarization lasting 100-200 ms before firing repetitively, whereas neurons without $I_{\mathrm{A}}$ discharge action potentials throughout the duration of the current pulse without a delay exceeding the membrane time constant (Fig. $2 B$ shows cycle-triggered averages of 5-10 sweeps to demonstrate the repeatability of delayed excitation or the lack thereof in type- 1 and type- 2 preBötC neurons).

The presence of $I_{\mathrm{h}}$ was determined by setting baseline $V_{\mathrm{m}}$ between -40 and $-50 \mathrm{mV}$ and delivering $400 \mathrm{~ms}$ negative current pulses of sufficient amplitude to hyperpolarize the neuron to -70 to $-90 \mathrm{mV}$, which is sufficient to evoke $I_{\mathrm{h}}$ if it is expressed by the neuron (Mironov et al., 2000; Thoby-Brisson et al., 2000). Neurons with $I_{\mathrm{h}}$ exhibit a "sag" depolarization of $\sim 10 \mathrm{mV}$ after being transiently hyperpolarized as well as a postinhibitory rebound after the negative current pulse terminates (Fig. $1 B$ shows cycle-triggered averages of 5-10 sweeps to demonstrate the repeatability of sag or the lack thereof in type- 1 and type- 2 preBötC neurons).

Among the rhythmic neurons recorded in our preBötC cultures, $57 \%(n=24)$ were type- 1 neurons and $31 \%(n=13)$ were type- 2
Because voltage-clamping inherently suffers from a lack of space clamp as a function of distance from the recording pipette, changes in membrane potential enforced at the soma via SEVC are attenuated at distal dendritic locations. The exact amount of that attenuation depends on passive cable properties (e.g., length, diameter, branch order) and density of voltage-gated conductances (Bar-Yehuda and Korngreen, 2008), but space-clamp error must be factored into data analysis and interpretation.

In type- 1 neurons, $I_{\mathrm{A}}$ activates at subthreshold membrane potentials and fully inactivates at membrane potentials less negative than $-40 \mathrm{mV}$ (Hayes et al., 2008). Therefore, neurons were first held at a command potential $\left(V_{\mathrm{C}}\right)$ of -75 to $-80 \mathrm{mV}$, where $I_{\mathrm{A}}$ is steady-state de-inactivated in the presence of TTX (Hayes et al., 2008). We then evoked $I_{\mathrm{A}}$ by $400 \mathrm{~ms}$ step commands to $+20 \mathrm{mV}$, which inactivated after $\sim 100-200 \mathrm{~ms}$ (Fig. 3A).

Dendritic $\mathrm{Ca}^{2+}$ transients were then imaged during delivery of fast (150 ms duration) positive-going voltage ramps, starting at $V_{\mathrm{C}}$ and increasing to a final membrane potential capable of activating voltage-gated $\mathrm{Ca}^{2+}$ currents (mean $=7.8 \pm 10.2 \mathrm{mV}, n=$ 9 ). These short-duration, rapidly increasing voltage ramps mimic the onset of endogenous inspiratory bursts and elicit suprathreshold $\mathrm{Ca}^{2+}$ responses within the transient phase of $I_{\mathrm{A}}$ activation (Fig. $3 B$ ). We then added $2 \mathrm{~mm} 4$-AP to the bath, confirmed blockade of $I_{\mathrm{A}}$ with voltage-clamp step commands (Fig. $3 \mathrm{~A}$ ), and then repeated the voltage-ramp command to remeasure the dendritic $\mathrm{Ca}^{2+}$ transients (Fig. 3B).

The command stimulus was constant between control and 4-AP sweeps for each recorded neuron, but the peak voltage am- 
A

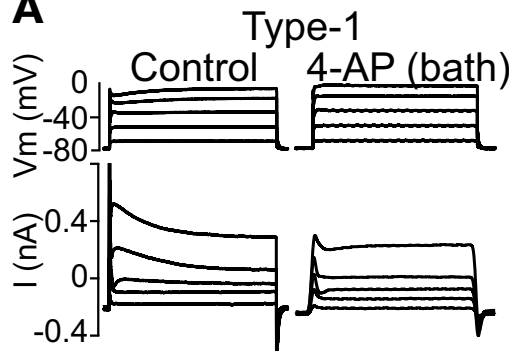

B

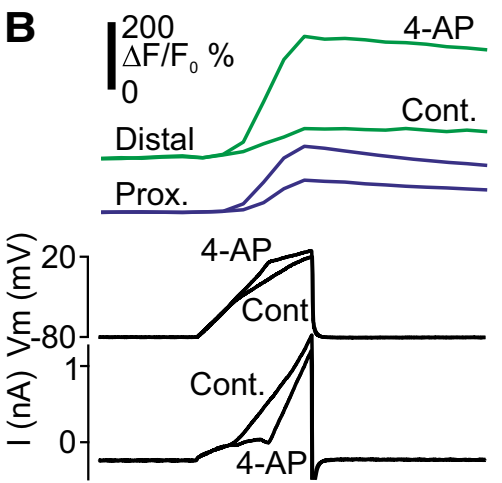

C

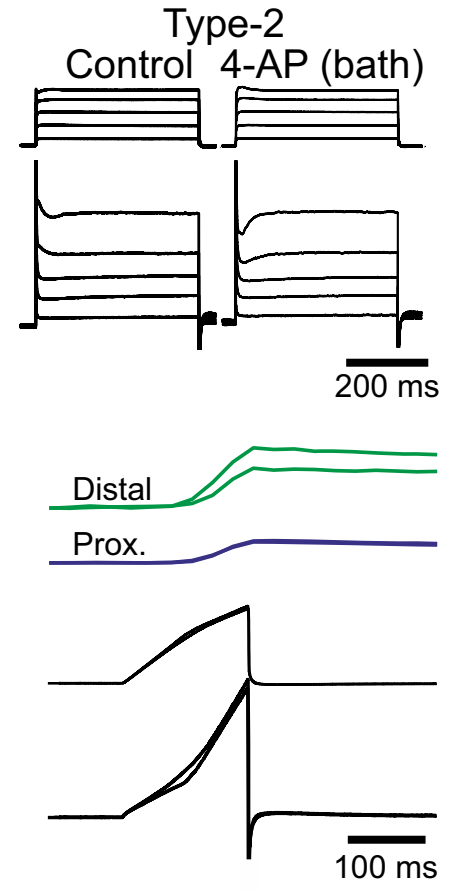

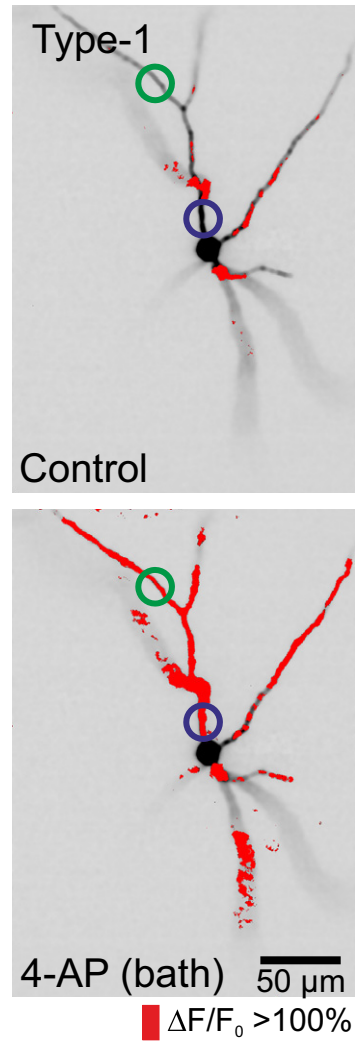
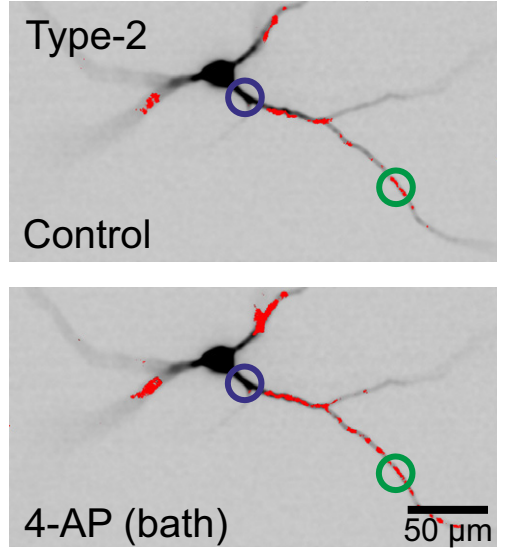

D

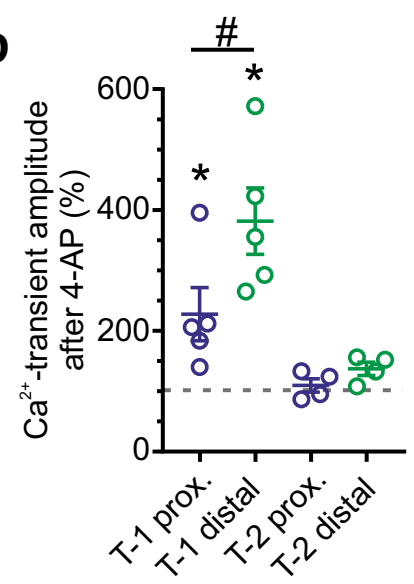

Figure 3. Bath applied 4-AP increase dendritic $\mathrm{Ca}^{2+}$ transients in response to ramp depolarizations. $\boldsymbol{A}$, Voltage-clamp traces, with TTX (1 $\mu \mathrm{M})$ present in the ACSF, before and after adding $2 \mathrm{~mm}$ 4-AP to the bath. Left-most neuron shows a transient outward current, which is blocked by 4-AP classifying the neuron as a type-1. Right-most neuron shows no evidence out a transient outward current, classifying the neuron as a type-2. $\boldsymbol{B}, \mathrm{Ca}^{2+}$ transients in proximal and distal dendritic compartments in the two neurons in response to a $150 \mathrm{~ms}$ voltage ramp ( -80 to $20 \mathrm{mV}$ ), and associated voltage and current traces. Responses before and after adding $2 \mathrm{~mm} 4-\mathrm{AP}$ to the bath is overlaid. Blue lines, Proximal dendritic compartments; green lines, distal dendritic compartments. C, Live morphology (AlexaFluor 594 hydrazide present in the patch solution) of the two neurons with thresholded dendritic $\mathrm{Ca}^{2+}$ transient amplitude overlaid in red. Red indicates $\mathrm{Ca}^{2+}$ transient amplitudes $\left(\Delta F / F_{0}\right)>100 \%$. $D$, Group data (mean $\left.\pm S E M\right)$

plitude achieved by the stimulus waveform (which is measurable in SEVC) nevertheless increased across all neurons after addition of 4-AP because of increased effectiveness of the SEVC (type-1: $8.5 \pm 2.2 \%, \mathrm{n}=5$; type-2: $2.0 \pm 1.5 \%$; $n=4)$. Changes in peak amplitude of the stimulus waveform were significantly greater in type-1 neurons than those in type- 2 neurons ( $n=5$ type- $1, n=4$ type-2 neurons; $t_{(7)}=4.93, p=0.0017$, two-tailed unpaired $t$ test). Input resistance in each cell was measured by taking the slope of the $I-V$ curve from the first $20 \mathrm{mV}$ of increase above command potential; a range in which no apparent active conductances were activated. Mean input resistance was $221 \pm 97 \mathrm{M} \Omega$ in type- 1 neurons and $181 \pm 77 \mathrm{M} \Omega$ in type- 2 neurons, which was a negligible difference $(n=$ 5 type- $1, n=4$ type- 2 neurons; $t_{(7)}=0.68$, $p=0.52$, two-tailed unpaired $t$ test).

To visualize how voltage propagation through dendritic compartments is affected by a blockade of $I_{\mathrm{A}}$, we measured the relative increase in $\mathrm{Ca}^{2+}$ indicator fluorescence $\left(\Delta F / F_{0}\right)$ evoked by voltage ramps before and after exposure to 4-AP (Fig. 3C,D). Proximal dendritic compartments ( $\leq 33 \mu \mathrm{m}$ from the soma) and distal dendritic compartments ( $\geq 69 \mu \mathrm{m}$ from the soma) were sampled with a minimum distance between measurement sites of $54 \mu \mathrm{m}$. The mean proximal ROI distance from the soma was $14 \pm 9 \mu \mathrm{m}$ $(n=9)$ and the mean distal ROI distance from the soma was $101 \pm 21 \mu \mathrm{m}(n=9)$. Among type-1 neurons, the amplitude of fluorescent $\mathrm{Ca}^{2+}$ transients elicited by voltage ramps increased significantly by $228 \pm 98 \%$ at proximal regions after addition of 4-AP (Fig. 3D; $\Delta F / F_{0}$ before vs $\Delta F / F_{0}$ after 4 -AP, $n=5$ type- 1 neurons; $t_{(4)}=2.40, p=0.037$, one-tailed paired $t$ test). The amplitude of transients measured at distal dendritic compartments also increased significantly by $382 \pm$ $123 \%\left(\Delta F / F_{0}\right.$ before vs $\Delta F / F_{0}$ after $4-\mathrm{AP}$, $n=5$ type- 1 neurons; $t_{(4)}=2.87, p=$ 0.022 , one-tailed paired $t$ test). The observed increase in response amplitude at distal regions in type-1 neurons was significantly greater than proximal regions (normalized 4-AP effect proximal vs normalized 4-AP effect distal, $n=5$ type- 1

expressing the relative $\mathrm{Ca}^{2+}$-transient amplitude after 4-APfrom a sample of type- $1(\mathrm{~T}-1 ; n=6)$ and type-2 $(\mathrm{T}-2 ; n=5)$ neurons. Note that both proximal and distal dendritic regions of type- 1 neurons show a larger $\mathrm{Ca}^{2+}$ transient amplitude after 4-AP $(*)$, and that distal regions show a larger increase than proximal regions (\#). 


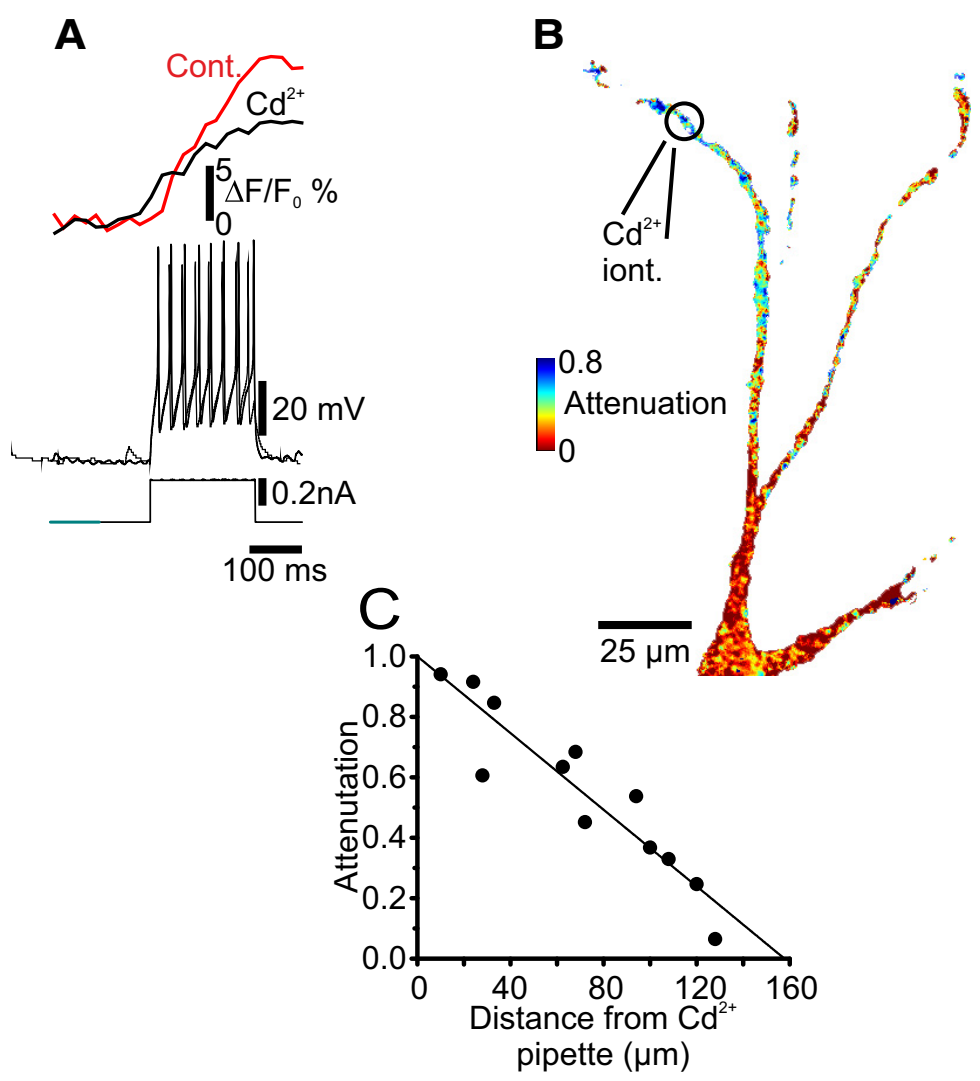

Figure 4. Dendritic iontophoresis of $\mathrm{Cd}^{2+}$ reduce dendritic $\mathrm{Ca}^{2+}$-transients evoked by current pulses. $\boldsymbol{A}$, Two hundred millisecond current pulses applied to a type-1 neuron evoking a spike train, and the resulting $\mathrm{Ca}^{2+}$ transient in a dendritic compartment (red trace, $\sim 130 \mu \mathrm{m}$ from soma, at the $\mathrm{Cd}^{2+}$ application site). Iontophoresis of $\mathrm{Cd}^{2+}(2 \mathrm{~min}, 500 \mathrm{~ms}$ pules, $1 \mathrm{~Hz})$ reduced the dendritic $\mathrm{Ca}^{2+}$ transient (black trace). $\boldsymbol{B}$, Same neuron as in $\boldsymbol{A}$, showing the spatial distribution of the $\mathrm{Cd}^{2+}$ effect, expressed as attenuation (red: 0 to blue: 0.8 ). Note that the site of application (pipette insert) has the largest attenuation compared with more proximal sites along the dendrite. $C$, Group data $(n=7)$ showing the attenuation of spike-train evoked $\mathrm{Ca}^{2+}$ transients in response to $\mathrm{Cd}^{2+}$ as a function of distance along the dendrite toward the soma. Black line is a linear fit.

neurons; $t_{(8)}=2.56, p=0.034$, two-tailed unpaired $t$ test), suggesting a nonlinear increase in the amplitude of $\mathrm{Ca}^{2+}$ influx between perisomatic and distal dendritic compartments.

Among type-2 neurons, the amplitude of fluorescent $\mathrm{Ca}^{2+}$ transients did not increase significantly at proximal regions $\left(\Delta F / F_{0}\right.$ before vs $\Delta F / F_{0}$ after 4 -AP, $n=4$ type-2 neurons, $t_{(3)}=0.46, p=$ 0.34 , one-tailed paired $t$ test), nor did the amplitude at distal regions $\left(\Delta F / F_{0}\right.$ before vs $\Delta F / F_{0}$ after 4 -AP, $n=4$ type- 2 neurons, $t_{(3)}=1.72, p=0.091$, one-tailed paired $t$ test). This suggests that $I_{\mathrm{A}}$ does not play a significant role in governing dendritic depolarization of type- 2 preBötC neurons, particularly compared with their counterparts the type- 1 preBötC neurons (Fig. $3 D$, compare columns 1,2 to 3,4 ).

These results demonstrate that a large increase in the voltage ramp-evoked $\mathrm{Ca}^{2+}$ transient occurs globally in type-1 neurons when 4-AP-sensitive currents are blocked (Fig. 3C; depicting raw $\Delta F / F_{0}$ values $>100 \%$ ), and that this increase is greatest at distal dendritic regions. The data imply either: (1) blocking $I_{\mathrm{A}}$ increases the length constant by removing $I_{\mathrm{A}}$-mediated shunting of the dendritic plasma membrane, which enhances the ability of somatic command potentials to depolarize the dendrite, or (2) blocking $I_{\mathrm{A}}$ augments $\mathrm{Ca}^{2+}$ channel activation at distal dendritic sites. In either scenario, our data cannot distinguish whether changes in the measured $\mathrm{Ca}^{2+}$ transient arose predominantly from diminution of somatic or dendritic $I_{\mathrm{A}}$ (or both). Thus, we sought to determine whether ionic membrane currents could be blocked on distal dendritic sites while minimally affecting the soma.

\section{Ion channels can be blocked in a spatially restricted manner via iontophoresis}

To demonstrate that ion channels can be blocked in morphologically selective regions of preBötC neurons in culture, we conducted a positive control experiment in which $\mathrm{Cd}^{2+}$ was applied focally via iontophoresis to block $\mathrm{Ca}^{2+}$ channels in distal dendrites (Fig. 4). Simultaneous whole-cell patch-clamp and $\mathrm{Ca}^{2+}$-imaging recordings were acquired from preBötC neurons in slice cultures. Spontaneous network activity was suppressed by reducing extracellular $\mathrm{K}^{+}$concentration from 8 to $2.5 \mathrm{~mm}$ and increasing extracellular $\mathrm{Ca}^{2+}$ concentration from 1.5 to $2 \mathrm{~mm}$. Membrane potential was held between -55 and $-60 \mathrm{mV}$ to prevent spontaneous spiking. A pipette containing an aqueous solution of $200 \mathrm{mM} \mathrm{CdSO}_{4}$ was positioned at distal dendritic regions. Positive current through the patch-recording pipette was then injected at the soma in either squarewave pulses (400 ms, 0.1-0.3 nA, $1 \mathrm{~Hz}$, causing repetitive spiking; Fig. $4 A$ ) or short repetitive current pulses ( $3 \mathrm{~ms}, 0.8$ $1.2 \mathrm{nA}, 100 \mathrm{~Hz}$ ) evoking brief trains of action potentials. The stimulus was maintained between control and $\mathrm{Cd}^{2+}$ application in each cell. After acquiring control sweeps, $\mathrm{Cd}^{2+}$ was applied to the dendrite using iontophoresis (400-500 ms, $1 \mathrm{~Hz}$, +5-10 nA), 30-60 s were allowed to pass for equilibration of drug ejection, and imaging sweeps were repeated. Peak $\Delta F / F_{0}$ values were sampled from two locations in each cell, separated by a minimum of $50 \mu \mathrm{m}$ ( $n=7$ neurons): proximal and distal dendritic regions as before, as well as the soma. The decrease in $\mathrm{Ca}^{2+}$ transient amplitude after application of $\mathrm{Cd}^{2+}$ was normalized to control sweeps.

Distance between the tip of the iontophoresis pipette and center of each ROI was measured in the $x-y$ plane. Because both the iontophoresis pipette and imaged cellular compartments occupy approximately the same focal plane, the difference in their positions estimated the distance in three-dimensional space between the point of drug application and the ROI. Regression analysis revealed a linear relationship between the degree of $\mathrm{Ca}^{2+}$ signal attenuation and straight-line distance from the pipette (Fig. $4 C$; $y$-intercept $=1.0, R^{2}=0.85$; ANOVA $F=64.45, p<0.001, n=$ $14)$. This model suggests that $<50 \%$ of the somatically evoked, dendritic $\mathrm{Ca}^{2+}$ transient is attenuated when the drug $\left(\mathrm{Cd}^{2+}\right.$ in this case) is applied $79 \mu \mathrm{m}$ away from the ROI. Although the mobility in the extracellular environment due to applied electric field undoubtedly differs between $\mathrm{Cd}^{2+}$ and 4-AP, both drugs are extracellular ion channel blockers that are not taken up by cellular processes. Thus, to ensure that recorded somata remained unaffected by iontophoretic drug application, we maintained a minimum of $96 \mu \mathrm{m}$ (average of $121 \pm 20 \mu \mathrm{m}, n=12$ experiments) between the point of drug application and the nearest edge of somatic compartments in all subsequent local drug application experiments. 
Dendritic $I_{\mathrm{A}}$ blockade increases $\mathrm{Ca}^{2+}$ response to somatically evoked stimuli in type-1 neurons

To determine whether 4-AP increases the $\mathrm{Ca}^{2+}$ response of type- 1 neurons by blocking dendritic $I_{\mathrm{A}}$, we applied 4-AP via iontophoresis to distal dendrite sites (Fig. 5; mean dendritic length from soma: $118 \pm$ $21 \mu \mathrm{m}, n=12$ ) in rhythmically active preBötC neurons and measured the amplitude of $\mathrm{Ca}^{2+}$ transients in response to trains of 10 action potentials triggered by somatic current injection. Whole-cell patch-clamp recordings of rhythmically active preBötC neurons were acquired and their membrane properties were tested in current-clamp recording mode to test for the presence of $I_{\mathrm{A}}$ and $I_{\mathrm{h}}$, and thus determine whether they were type- 1 or type-2. At least $40 \mathrm{~min}$ of whole-cell recording was allowed to elapse to allow for dye diffusion at distal compartments. All imaging sweeps were performed in the presence of $2.5 \mathrm{mM} \mathrm{K}^{+}$and $2 \mathrm{mM} \mathrm{Ca}^{2+}$, which suppresses respiratory rhythm.

We further added the ionotropic excitatory amino acid receptor antagonist NBQX $(10-20 \mu \mathrm{M})$ to the perfusate to suppress excitatory synaptic activity. In both control trials and local 4-AP application trials, ROIs were sampled from distal dendritic regions within $30 \mu \mathrm{m}$ of the iontophoresis pipette. To verify that the effect of 4-AP observed near the site of iontophoresis was caused by local ion channel blockade, we also sampled the relative change in fluorescence from a presumably unaffected compartment (i.e., other dendritic branches, proximal sites on the branch of drug application, and the soma) located at least $100 \mu \mathrm{m}$ away in the $x-y$ plane from the site of iontophoresis (mean distance: $139 \pm 32 \mu \mathrm{m}, n=12$ ). Pipettes containing $45 \mathrm{~mm} 4$-AP dissolved in saline (165 mM NaCl, 0.2\% dextran-TMR, pH 7.5) were positioned at distal dendritic sites. During drug application trials, we applied continuous ejection pulses as before $(400-500 \mathrm{~ms}$, $1 \mathrm{~Hz},+20-45 \mathrm{nA})$. In type- 1 neurons, spike train-evoked $\mathrm{Ca}^{2+}$ transients at distal dendritic sites (near the point of drug application) increased substantially to $155 \pm 29 \%$ (Fig. $5 A-C ; \Delta F / F_{0}$ before vs $\Delta F / F_{0}$ after 4 -AP; $n=9$ type- 1 neurons; $t_{(8)}=5.22, p=$ 0.0008 , two-tailed paired $t$ test) compared with control. Regions farther than $100 \mu \mathrm{m}$ away from the site of iontophoresis did not show an increase in $\Delta F / F_{0}$ compared with the control response $\left(\Delta F / F_{0}\right.$ before vs $\Delta F / F_{0}$ after 4 -AP; $n=9$ type- 1 neurons, $t_{(8)}=$ $2.08, p=0.071$, two-tailed paired $t$ test). The increase at sites near the point of iontophoresis was significantly greater than on other dendritic branches or the soma (normalized 4-AP effect proximal vs normalized 4 -AP effect distal; $n=9$ type- 1 neurons, $t_{(16)}=$ 5.65, $p=0.0001$, two-tailed unpaired $t$ test).

Type- 2 neurons did not show an increase in $\Delta \mathrm{F} / \mathrm{F}_{0}$ evoked by current pulses at the site of iontophoresis (Fig. $5 C ; \Delta F / F_{0}$ before vs $\Delta F / F_{0}$ after 4 -AP; $n=3$ type-2 neurons, $t_{(2)}=0.26, p=0.82$, two-tailed paired $t$ test) or at sites $>100 \mu \mathrm{m}$ from the site of

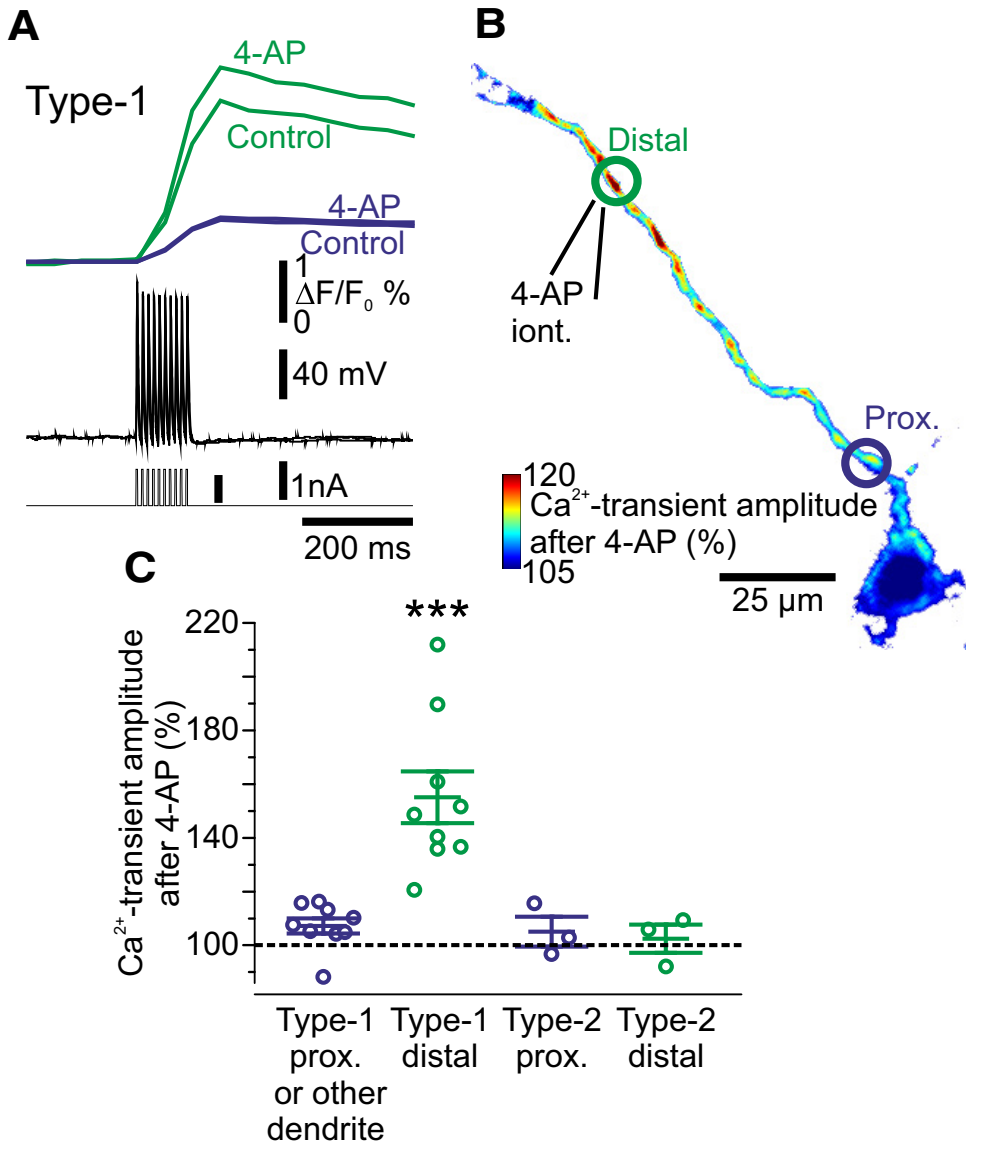

Figure 5. Dendritic iontophoresis of 4-AP increases dendritic $\mathrm{Ca}^{2+}$ transients evoked by current pulse trains. $\boldsymbol{A}, \mathrm{A}$ train of current pulses ( 10 pulses, 3 ms duration, $100 \mathrm{~Hz}$ ) applied to a type- 1 neuron evoking 10 spikes. The resulting $\mathrm{Ca}^{2+}$ transients in a dendritic compartment ( $\sim 110 \mu \mathrm{m}$ from soma, placed next to the 4-AP pipette) is shown above the spikes before, and after shown for another more proximal dendritic site close to soma (blue traces). $\boldsymbol{B}$, Same neuron as in $\boldsymbol{A}$, showing the color coded spatial 作 (pipette insert) has the largest increase in the $\mathrm{Ca}^{2+}$ transient compared with more proximal sites along the dendrite. Group data (mean \pm SEM) showing the $\mathrm{Ca}^{2+}$ transient amplitude after 4-AP application for proximal and distal (next to 4-AP pipette) sites in the two types of neurons ( $n=9$ type- 1 neurons; $n=3$ type- 2 neurons). Black dotted line is $100 \%$, i.e., no change. Note that distal dendritic sites in type-1 neuron show a large increase in $\mathrm{Ca}^{2+}$-transients after application of 4-AP( $\left.{ }^{* * *}\right)$.

iontophoresis $\left(\Delta F / F_{0}\right.$ before vs $\Delta F / F_{0}$ after 4 -AP; $n=3$ type-2 neurons, $t_{(2)}=0.18, p=0.87$, two-tailed paired $t$ test). These results demonstrate that blockade of $I_{\mathrm{A}}$ on dendrites has a substantial effect on the size of distal dendritic $\mathrm{Ca}^{2+}$ transients evoked by somatic current pulses in type- 1 preBötC neurons, but that $I_{\mathrm{A}}$ blockade has a negligible effect on distal dendritic $\mathrm{Ca}^{2+}$ transients evoked at the soma in type-2 neurons.

\section{$I_{\mathrm{A}}$ blockade increases dendritic depolarizing response to glutamate application in type- 1 neurons}

To better understand how the dendritic $I_{\mathrm{A}}$ might influence the synaptic integration in type- 1 neurons, we applied glutamate to distal dendritic sites of type- 1 neurons and recorded the resulting somatic depolarization before and after iontophoresis of 4-AP at the same site. These experiments were performed in $1 \mu \mathrm{M}$ TTX to preclude action-potential driven synaptic transmission not under our control. We used a dual-barrel theta pipette containing glutamate $(150 \mathrm{~mm})$ in one barrel and $4-\mathrm{AP}(50 \mathrm{~mm})$ in the other. The tip of the theta pipette was placed at dendritic sites that were $116-138 \mu \mathrm{m}$ from the soma $(n=4$ neurons in 4 different cultures). Short repetitive $(100 \mathrm{~ms}, 0.2 \mathrm{~Hz})$ puffs of glutamate at 
A

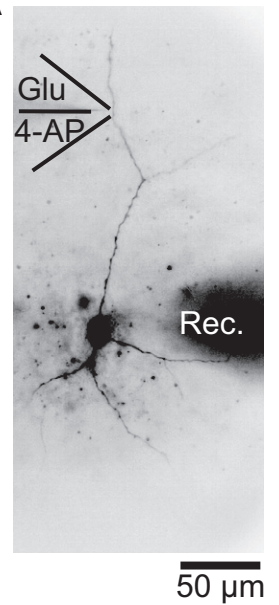

B

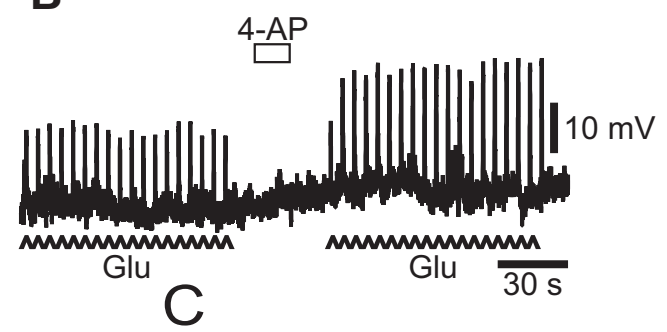

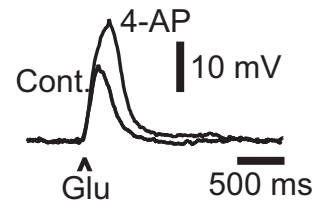

Figure 6. The amplitude and width of dendritic glutamate responses are increased by dendritic iontophoresis of 4-AP in type-1 neurons. $A$, Morphology of a live type-1 neuron (AlexaFluor 594 hydrazide present in the patch solution) with a somatic recording pipette (Rec), and a dendritically placed (138 $\mu \mathrm{m}$ from soma) theta-iontophoresis pipette containing glutamate in one barrel and 4-AP in the other (Glu/4-AP). B, Repetitive 100-ms-duration iontophoretic pulses of glutamate in the presence of bath-applied TTX before (Cont) and after iontophoresis of 4-AP (20 s). C, Overlaid traces of the glutamate response before (Cont) and after 4-AP iontophoresis (4-AP). Note the increase in amplitude and width of the glutamate response.

dendritic sites gave rise to evoked depolarizations recorded at the soma (total duration $\sim 500 \mathrm{~ms}$ or less, half-amplitude width $90-$ $244 \mathrm{~ms}$; maximum amplitude $5-15 \mathrm{mV}$; Fig. $6 \mathrm{~B}, C$ ). We inhibited A-current on dendrites at the site of glutamate application via $10-20 \mathrm{~s}$ of 4-AP iontophoresis. Upon resuming pulsed glutamate delivery, we recorded a $149 \pm 14 \%$ increase in amplitude and $160 \pm 42 \%$ increase in half-amplitude width of the glutamate responses recorded at the soma (Fig. $6 C ; n=4$ type- 1 neurons, $t_{(3)}=2.74, p=0.036$, and $t_{(3)}=4.22, p=0.012$, one-tailed paired $t$ test).

\section{Discussion}

The subcellular distribution of active membrane currents in preBötC neurons is largely unknown. Here we show that an $I_{\mathrm{A}}$-like current is expressed on the dendrites of type- 1 preBötC neurons, which can influence synaptic integration and thus inspiratory rhythm- and burst generation.

$I_{\mathrm{A}}$ in type-1 preBötC neurons has previously been posited to affect the onset of the inspiratory burst phase during rhythmic network activity. A complete network-wide blockade of $I_{\mathrm{A}}$ causes disordered inspiratory rhythms (Hayes et al., 2008). These authors measured $I_{\mathrm{A}}$ in somatic whole-cell and outside-out patches, which cannot provide information about its subcellular distribution. However, the distribution of $I_{\mathrm{A}}$ could influence the integrative properties of rhythmically active neurons. Dendrites expressing $I_{\mathrm{A}}$ can selectively promote and amplify responses to network activity that is synchronized while inhibiting postsynaptic responsiveness to asynchronous and sparse spontaneous input.

Minute inhibition of 4-AP-sensitive currents has a strong effect on network excitability. Network activity responds even to doses of 4 -AP $(50 \mu \mathrm{M})$ that are 40 -fold less than the reported $\mathrm{IC}_{50}$ for $I_{\mathrm{A}}(\sim 2 \mathrm{mM})$ in acute slices containing the preBötC (Hayes et al., 2008). Attenuation of $I_{\mathrm{A}}$ appears to advance the phase of the inspiratory cycle, nearly doubling the rhythm frequency. Attenuation of $I_{\mathrm{A}}$ also increases the amplitude of neural bursts in regions dorsomedial to the preBötC, which likely arises from inspiratory-related hypoglossal motoneurons. Last, attenuation of $I_{\mathrm{A}}$ lengthens the duration of somatic and dendritic $\mathrm{Ca}^{2+}$ transients in preBötC neurons. Although 4-AP can inhibit delayed

rectifier current, conceivably accounting for an overall increase in excitability, we suspect that these off-target effects are negligible at such low $(50 \mu \mathrm{M})$ doses of 4-AP. Therefore, a more parsimonious explanation for these data is that partial blockade of $I_{\mathrm{A}}$ diminishes its ability to gate burst amplifying currents during the preinspiratory phase.

Increased activity in dorsomedial areas outside the preBötC suggests three possibilities: (1) activity in the preBötC is augmented, (2) preBötC activity propagates more readily to downstream areas, or (3) that excitability is increased in dorsomedial premotor or motor pools. $\mathrm{Ca}^{2+}$ activity measured in neurons within the preBötC suggests local network activity may indeed be augmented. $\mathrm{Ca}^{2+}$ transients in both somata and the neuropil (dominated by dendrites) lasted longer and decayed more slowly following a $50 \mu \mathrm{M}$ dose of 4-AP, demonstrating that burst activity at the systems level is prolonged when $I_{\mathrm{A}}$ is partially blocked.

We failed to pharmacologically characterize the $I_{\mathrm{A}}$-like current present in the preBötC after using three different channelspecific Kv4 inhibitors; the compounds did not affect rhythmic output. Thus, pharmacological specificity and molecular identification of the channel types giving rise to $I_{\mathrm{A}}$ in the preBötC remains an issue. Transcriptomic data show that preBötC neurons sharing a common lineage with type-1 neurons (i.e., Dbx1derived cells) differentially and robustly express Kv4.2 with KChIP2 (Hayes et al., 2017), forming $I_{\mathrm{A}}$-like channels that favor non-inactivated states with relatively fast de-inactivation kinetics (Amadi et al., 2007). The transcriptome study catalogs all $\mathrm{K}^{+}$ channels in rhythmogenic and non-rhythmogenic preBötC neurons (Hayes et al., 2017). Specific targeting of these channel types (using genetic rather than pharmacological tools alone) will be necessary to assess the rhythmogenic role of $I_{\mathrm{A}}$.

To determine what $I_{\mathrm{A}}$ might be doing at the cellular level, we needed a means of interrogating activity in the dendrites of rhythmically active preBötC neurons. Organotypic slice cultures containing the preBötC flatten out and become transparent over several days, which improves the signal-to-noise ratio of $\mathrm{Ca}^{2+}$ fluorescence dendritic imaging. To better understand how type- 1 and type- 2 neurons integrate synaptic input, we then asked whether $I_{\mathrm{A}}$ in rhythmically active neurons exists on dendrites and whether it has a significant impact on the ability for voltage to spread between somatic and distal dendritic compartments.

Blockade of $I_{\mathrm{A}}$ in type- 1 neurons results in a $\sim 130 \%$ increase in the amplitude of ramp-evoked $\mathrm{Ca}^{2+}$ transients at proximal dendritic regions and a $\sim 280 \%$ increase at distal dendritic regions. In contrast, type- 2 neurons show no significant increase at proximal or distal dendritic regions after bath application of 4 -AP. Global increases in the $\mathrm{Ca}^{2+}$ transients of type- 1 neurons may reflect a change in the electrotonic compactness of the neuron: more of the somatically triggered depolarization propagates from the soma to the distal dendrite. Alternatively, depolarization triggered at the soma might be less counteracted by $I_{\mathrm{A}}$ before passively propagating along dendrites. 
Bath application of 4 -AP mediated a $\sim 2-8 \%$ rise in the maximum amplitude of delivered voltage ramps in both type- 1 and type-2 neurons, signifying a change in the strength of the SEVC. By some immeasurable degree, the consistent increase in ramp amplitudes definitely contributes to the observed change in evoked transients on both proximal and distal dendrites. The strength of the SEVC, and by extension the ability to increase the membrane potential of the neuron, is thus correlated with the presence or absence of $I_{\mathrm{A}}$, supporting the hypothesis that it affects either electrotonic compactness or resists membrane depolarization. The data from these experiments is unable to distinguish whether the apparent increase in electrotonic compactness, or reduced inhibition of membrane depolarization, is the result of solely somatic $I_{\mathrm{A}}$ or additionally includes dendritic $I_{\mathrm{A}}$. To definitively determine whether the increases in the amplitude of ramp-evoked $\mathrm{Ca}^{2+}$ transients were caused by dendritic $I_{\mathrm{A}}$ in conjunction with somatic $I_{\mathrm{A}}$, we attempted to locally block $I_{\mathrm{A}}$ on dendrites of rhythmically active neurons.

Spike train-evoked $\mathrm{Ca}^{2+}$ fluorescence increased by $\sim 155 \%$ at local sites of 4-AP iontophoresis, with no change in fluorescence in other compartments located either proximally on the same parent branch, at the soma, or on other dendritic branches occupying the same focal plane. Thus, these results indeed suggest that the density of ion channels giving rise to $I_{\mathrm{A}}$ in type- 1 preBötC neurons extends well beyond the soma into distal dendritic compartments. It is important to distinguish that increased dendritic $\mathrm{Ca}^{2+}$ influx in the presence of 4-AP does not necessarily map one-to-one with a rise in voltage, but is generally indicative of changes in voltage. The nonlinear increase in evoked $\mathrm{Ca}^{2+}$ transients at distal dendritic sites can be equally interpreted as a reduction in voltage decay between the soma and dendrites or as the recruitment of previously inhibited inward currents. Considering the overall global increase in $\mathrm{Ca}^{2+}$ response, $I_{\mathrm{A}}$ at minimum alters electrotonic compactness since all cellular compartments appear to charge in response to voltage stimuli more effectively.

Finally, we show that dendritic $I_{\mathrm{A}}$ blockade increases the amplitude of glutamate-evoked postsynaptic depolarizations recorded at the soma of type-1 neurons (Fig. 6). Small-amplitude glutamate responses $(5-15 \mathrm{mV})$ triggered at distal dendritic sites became augmented when nearby dendritic $I_{\mathrm{A}}$ was blocked. Thus, $I_{\mathrm{A}}$ demonstrably dampens small-amplitude dendritic input.

The data suggest that $I_{\mathrm{A}}$ on the dendrites can counteract sparse excitatory synaptic events in preBötC neurons. Nevertheless, during oscillatory activity in the preBötC, $I_{\mathrm{A}}$ would inactivate via temporally summating excitatory inputs lasting $200 \mathrm{~ms}$ (or more). Preinspiratory activity of type-1 neurons exceeds $400 \mathrm{~ms}$ (Rekling et al., 1996), indicating that building recurrent excitation ordinarily outlasts the transient phase of $I_{\mathrm{A}}$, which would allow steady-state inactivation to occur during a typical inspiratory cycle in type-1 neurons. (Hayes et al., 2008) estimated the contribution of $I_{\mathrm{A}}$ to the currents flowing in preBötC neurons during an inspiratory burst, and found that $I_{\mathrm{A}}$ rapidly achieves maximal activation, diminished throughout the burst, and carries very little current during the inter-inspiratory burst interval. With $I_{\mathrm{A}}$ unavailable due to inactivation, type- 1 neurons may transition from a relatively low-excitability state, in which excitatory synaptic input is presumably inhibited by $I_{\mathrm{A}}$, to a high-excitability state that is more electrotonically compact and where amplifying currents, such as $I_{\text {CAN }}$ are more easily recruited. It would also explain why preinspiratory activity in type- 1 neurons become significantly shorter and rise more rapidly after network-wide block of $I_{\mathrm{A}}$ in acute slices (Hayes et al., 2008). This type of activity-dependent integration emphasizes why type- 1 neurons expressing $I_{\mathrm{A}}$ may be most critical in dictating the appropriately timed onset of inspiratory burst cycles.

In conclusion, $I_{\mathrm{A}}$ is dendritically expressed in type- 1 preBötC neurons, which ordinarily diminishes excitatory synaptic input. However, the shunting role of $I_{\mathrm{A}}$ can be relieved by steady-state inactivation. Apparent changes in electrotonic compactness after blockade of $I_{\mathrm{A}}$ support the notion that $I_{\mathrm{A}}$ decreases the length constant of dendrites and shunts incoming synaptic potentials, minimizing their influence on somatic voltage trajectory. During rhythmic network activity, steady-state inactivation of dendritic $I_{\mathrm{A}}$ could be achieved via building recurrent excitation during the preinspiratory phase of the inspiratory cycle. $I_{\mathrm{A}}$ in the dendrites of type-1 neurons could thus limit the effects of synaptic inputs that arrive asynchronously or sporadically. When activity in this recurrently connected network accumulates during each respiratory cycle, $I_{\mathrm{A}}$ likely inactivates, promoting depolarization and subsequent burst generation. Being colocalized at both the site of excitatory input and postsynaptic drive amplification (i.e., dendrites), $I_{\mathrm{A}}$ is thus able to integrate the state of the network as it stochastically builds in tonic excitability during recurrent excitation. A robust amplification of synaptic drive will occur only when network activity reaches a degree of persistent coincident activity capable of inactivating $I_{\mathrm{A}}$.

\section{References}

Amadi CC, Brust RD, Skerritt MR, Campbell DL (2007) Regulation of $\mathrm{Kv} 4.3$ closed state inactivation and recovery by extracellular potassium and intracellular KChIP2b. Channels 1:305-314. CrossRef Medline

Bar-Yehuda D, Korngreen A (2008) Space-clamp problems when voltage clamping neurons expressing voltage-gated conductances. J Neurophysiol 99:1127-1136. CrossRef Medline

Carroll MS, Ramirez JM (2013) Cycle-by-cycle assembly of respiratory network activity is dynamic and stochastic. J Neurophysiol 109:296-305. CrossRef Medline

Diochot S, Drici MD, Moinier D, Fink M, Lazdunski M (1999) Effects of phrixotoxins on the Kv4 family of potassium channels and implications for the role of Itol in cardiac electrogenesis. Br J Pharmacol 126:251-263. CrossRef Medline

Feldman JL, Del Negro CA (2006) Looking for inspiration: new perspectives on respiratory rhythm. Nat Rev Neurosci 7:232-242. CrossRef Medline

Feldman JL, Kam K (2015) Facing the challenge of mammalian neural microcircuits: taking a few breaths may help. J Physiol 593:3-23. CrossRef Medline

Feldman JL, Del Negro CA, Gray PA (2013) Understanding the rhythm of breathing: so near, yet so far. Annu Rev Physiol 75:423-452. CrossRef Medline

Forsberg D, Horn Z, Tserga E, Smedler E, Silberberg G, Shvarev Y, Kaila K, Uhlén P, Herlenius E (2016) $\mathrm{CO}_{2}$-evoked release of PGE2 modulates sighs and inspiration as demonstrated in brainstem organotypic culture. eLife 5:e14170. CrossRef Medline

Funk GD, Greer JJ (2013) The rhythmic, transverse medullary slice preparation in respiratory neurobiology: contributions and caveats. Respir Physiol Neurobiol 186:236-253. CrossRef Medline

Hayes JA, Mendenhall JL, Brush BR, Del Negro CA (2008) 4-Aminopyridinesensitive outward currents in preBötzinger complex neurons influence respiratory rhythm generation in neonatal mice. J Physiol 586:1921-1936. CrossRef Medline

Hayes JA, Kottick A, Picardo MCD, Halleran AD, Smith RD, Smith GD, Saha MS, Del Negro CA (2017) Transcriptome of neonatal preBötzinger complex neurones in Dbx1 reporter mice. Sci Rep 7:8669. CrossRef Medline

Hoffman DA, Magee JC, Colbert CM, Johnston D (1997) K+ channel regulation of signal propagation in dendrites of hippocampal pyramidal neurons. Nature 387:869-875. CrossRef Medline

Kam K, Worrell JW, Ventalon C, Emiliani V, Feldman JL (2013) Emergence of population bursts from simultaneous activation of small subsets of preBötzinger complex inspiratory neurons. J Neurosci 33:3332-3338. CrossRef Medline

Kampa BM, Stuart GJ (2006) Calcium spikes in basal dendrites of layer 5 
pyramidal neurons during action potential bursts. J Neurosci 26:74247432. CrossRef Medline

Klenk KC, Tenenholz TC, Matteson DR, Rogowski RS, Blaustein MP, Weber DJ (2000) Structural and functional differences of two toxins from the scorpion Pandinus imperator. Proteins 38:441-449. CrossRef Medline

Maffie JK, Dvoretskova E, Bougis PE, Martin-Eauclaire MF, Rudy B (2013) Dipeptidyl-peptidase-like-proteins confer high sensitivity to the scorpion toxin AmmTX3 to Kv4-mediated A-type K+ channels. J Physiol 591: 2419-2427. CrossRef Medline

Magee J, Hoffman D, Colbert C, Johnston D (1998) Electrical and calcium signaling in dendrites of hippocampal pyramidal neurons. Annu Rev Physiol 60:327-346. CrossRef Medline

Mironov SL (2008) Metabotropic glutamate receptors activate dendritic calcium waves and TRPM channels which drive rhythmic respiratory patterns in mice. J Physiol 586:2277-2291. CrossRef Medline

Mironov SL, Skorova EY (2011) Stimulation of bursting in pre-Bötzinger neurons by Epac through calcium release and modulation of TRPM4 and K-ATP channels. J Neurochem 117:295-308. CrossRef Medline

Mironov SL, Langohr K, Richter DW (2000) Hyperpolarization-activated current, $I_{\mathrm{h}}$, in inspiratory brainstem neurons and its inhibition by hypoxia. Eur J Neurosci 12:520-526. CrossRef Medline

Newell DW, Malouf AT, Franck JE (1990) Glutamate-mediated selective vulnerability to ischemia is present in organotypic cultures of hippocampus. Neurosci Lett 116:325-330. CrossRef Medline

Otsu Y, Marcaggi P, Feltz A, Isope P, Kollo M, Nusser Z, Mathieu B, Kano M, Tsujita M, Sakimura K, Dieudonné S (2014) Activity-dependent gating of calcium spikes by A-type $\mathrm{K}+$ channels controls climbing fiber signaling in purkinje cell dendrites. Neuron 84:137-151. CrossRef Medline

Pace RW, Del Negro CA (2008) AMPA and metabotropic glutamate receptors cooperatively generate inspiratory-like depolarization in mouse respiratory neurons in vitro. Eur J Neurosci 28:2434-2442. CrossRef Medline

Pace RW, Mackay DD, Feldman JL, Del Negro CA (2007) Inspiratory bursts in the preBötzinger complex depend on a calcium-activated non-specific cation current linked to glutamate receptors in neonatal mice. J Physiol 582:113-125. CrossRef Medline
Panaitescu B, Ruangkittisakul A, Ballanyi K (2009) Silencing by raised extracellular $\mathrm{Ca}^{2+}$ of pre-Bötzinger complex neurons in newborn rat brainstem slices without change of membrane potential or input resistance. Neurosci Lett 456:25-29. CrossRef Medline

Phillips WS, Herly M, Del Negro CA, Rekling JC (2016) Organotypic slice cultures containing the preBötzinger complex generate respiratory-like rhythms. J Neurophysiol 115:1063-1070. CrossRef Medline

Picardo MC, Weragalaarachchi KT, Akins VT, Del Negro CA (2013) Physiological and morphological properties of Dbx1-derived respiratory neurons in the pre-Bötzinger complex of neonatal mice. J Physiol 591: 2687-2703. CrossRef Medline

Rekling JC, Champagnat J, Denavit-Saubié M (1996) Electroresponsive properties and membrane potential trajectories of three types of inspiratory neurons in the newborn mouse brain stem in vitro. J Neurophysiol 75:795-810. CrossRef Medline

Ruangkittisakul A, Panaitescu B, Ballanyi K (2011) $\mathrm{K}^{+}$and $\mathrm{Ca}^{2+}$ dependence of inspiratory-related rhythm in novel "calibrated" mouse brainstem slices. Respir Physiol Neurobiol 175:37-48. CrossRef Medline

Ruangkittisakul A, Kottick A, Picardo MC, Ballanyi K, Del Negro CA (2014) Identification of the pre-Bötzinger complex inspiratory center in calibrated "sandwich" slices from newborn mice with fluorescent Dbx1 interneurons. Physiol Rep 2:e12111. CrossRef Medline

Smith JC, Greer JJ, Liu GS, Feldman JL (1990) Neural mechanisms generating respiratory pattern in mammalian brain stem-spinal cord in vitro: I. spatiotemporal patterns of motor and medullary neuron activity. J Neurophysiol 64:1149-1169. CrossRef Medline

Smith JC, Ellenberger HH, Ballanyi K, Richter DW, Feldman JL (1991) PreBötzinger complex: a brainstem region that may generate respiratory rhythm in mammals. Science 254:726-729. CrossRef Medline

Thoby-Brisson M, Telgkamp P, Ramirez JM (2000) The role of the hyperpolarization-activated current in modulating rhythmic activity in the isolated respiratory network of mice. J Neurosci 20:2994-3005. Medline

Yasuda R, Nimchinsky EA, Scheuss V, Pologruto TA, Oertner TG, Sabatini BL, Svoboda K (2004) Imaging calcium concentration dynamics in small neuronal compartments. Sci STKE 2004:pl5. CrossRef Medline 